$$
\text { DUE/PC/GC'183-T6 }
$$

\title{
Dense Inclined Flows: Theory and Experiments
}

Quarterly Technical Progress Report (October 1, 1992 to December 31, 1992)

Contract number: DE-AC22-91PC90183

Contract Period of Performance: January 1, 1991 to December 31, 1994

Contractor: Cornell University, Ithaca, NY 14853

Contract Participants: James T. Jenkins, Michel Y. Louge DOE/PC/90183--T6

DOE Project Manager: William C. Peters

DE93 011452

US/DOE patent clearance is not required prior to the publication of this document.

\section{Summary of accomplishments}

Rapid, gravity-driven flows of granular materials down inclines pose a challenge to our understanding. Even in situations in which the flow is steady and two-dimensional, the details of how momentum and energy are balanced within the flow and at the bottom boundary are not well understood. Thus we have undertaken a research program integrating theory, computer simulation, and experiment that will focus on dense entry flows down inclines. Its goal is to understand the regime of inclined flow that involves a large, relatively passive mass of graiıular material moving above a narrow region of intensely sheared, colliding grains. The effort involves the development of theory informed by the results of simultaneous computer simulations and the construction, instrumentation, and use of an experimental facility in which the variables necessary to assess the success or failure of the theory can be measured.

In the present reporting period, we have completed a series of experiments to measure the collision properties of various small particles. Results for $3 \mathrm{~mm}$ glass beads are presented here.

In addition, we have made progress in the design of a new sensor to measure simultaneously the particle volume fraction near the wall and the normal force of impact from individual collisions.

Finally, we have conducted additional simulations of the rapid granular flows of spheres interacting with a flat, frictional wall. The objective was to generate semi-empirical expressions for the boundary conditions imposed by the wall on the flow.

During the present reporting period, J. Jenkins has worked with the University of Florida and the Scripps Institute.

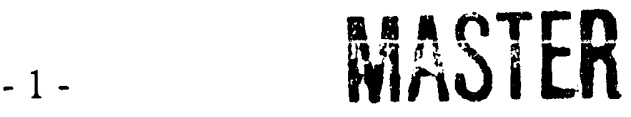




\section{Collision properties}

In this work, we measure the properties of the binary collisions between two small spheres in a free fall. The experimental apparatus was described in earlier reports. The collisions are described in terms of a coefficient of restitution e, a coefficient of Coulomb friction $\mu$, and a coefficient of tangential restitution $\beta_{0}$ [J.T. Jenkins, J. Applied Mechanics 59, 120-127 (1991)].

The coefficient e characterizes the incomplete restitution of the normal velocity at the point of contact,

$\mathbf{g}^{*} \cdot \mathbf{n}=-$ e $\mathbf{g} \cdot \mathbf{n} ;$

the coefficient of Coulomb friction $\mu$ relates the tangential and normal components of the impulse,

$|\mathbf{J} . \mathbf{t}|=\mu \mathbf{J} . \mathbf{n} ;$

and $\beta_{0}$ expresses the restitution of tangential velocity for contacts that do not involve sliding,

$g^{*} \cdot t=-\beta_{0}$ g.t.

At contact, $\mathbf{n}$ is the unit vector joining the centers of the two spheres, $t$ is a unit vector tangent to the spheres in the collision plane, $g$ is the relative velocity at the point of contact, and $\mathbf{J}$ is the impulse. The asterisk indicates values immediately following the collision.

The apparatus releases the two spheres in sequence without spin. From a stroboscopic photograph of the region near the collision, we infer the pre- and postcollision velocities of the spheres and their relative orientation upon contact. Measurements with slightly non-spherical particles typically exhibit greater scatter than perfect spheres. The experiments indicate that collisions between two glass spheres are adequately described by the model based on equations (1-3).

Figure 1 plots the ratio $\psi_{2} \equiv-\mathrm{g}^{*} . \mathbf{t} / \mathrm{g} . \mathrm{n}$ in terms of $\psi_{1} \equiv-$ g.t $/$ g.n for glass spheres of $3 \mathrm{~mm}$ diameter. When the spheres experience a sliding contact,

$\psi_{2}=\psi_{1}-\frac{7}{2} \mu(1+\mathrm{e}) \operatorname{sign}($ g.t $)$;

and when they slide,

$\psi_{2}=-\beta_{0} \psi_{1}$.

For $3 \mathrm{~mm}$ glass beads, we find $\beta_{0}=0.44, \mathrm{e}=0.93$ and $\mu=0.09$. 


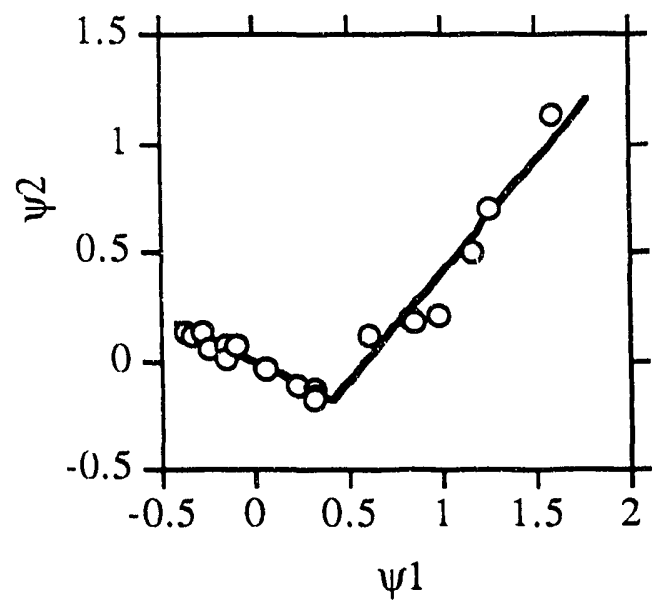

Fig. 1. Characteristic behavior of collisions described by Eqs. (1-3).

\section{Collision sensor}

We have designed a new sensor that will measure simultaneously the particle volume fraction near the bottom wall of the chute and the impulse exerted by individual particle collisions on a small surface located at the center of the probe. The volume fraction is recorded by a capacitance probe. The center of the probe is fitted with a Kistler load cell that records impulses arising from velocities as small as $0.2 \mathrm{~mm} / \mathrm{sec}$ for $3 \mathrm{~mm}$ glass particles.

\section{Simulations}

During the present reporting period, we have resumed our simulations on the interaction between a flat, frictional wall and rapid granular flows of frictional spheres. In particular, we have developed semi-empirical fits for the effective friction at the wall (ratio $\mathrm{S} / \mathrm{N}$ of shear to normal stress) and the flux of granular temperature through the wall. The following expressions are least-squares fits to the results of the simulations. They are valid for the range $0.5 \leq e \leq 1,0.1 \leq \mu \leq 0.4$, and $0 \leq \beta_{0} \leq 0.6$. We find for the effective friction, $\frac{\mathrm{S}}{\mathrm{N}} \approx \mu\left[1-\exp \left(-\frac{\mathrm{ar}}{\mu}\right)\right]$,

where

$\mathrm{a} \approx 0.35 \mathrm{e}^{-1.04(1-\mathrm{e})}\left[1-\mathrm{e}^{-4.45 \mu}\right]\left(1+\beta_{0}\right)^{\alpha}$,

and $\mathrm{r} \equiv \mathrm{g} / \sqrt{3 \mathrm{~T}}$ is the dimensionless slip. In (7), the exponent $\alpha$ is given approximately by $\alpha \approx 1-e^{-2.25 \mu}$ 
In these expressions, $g$ is the average relative velocity of the point of contact of the particle and the wall, and $\mathrm{T}$ is the translational granular temperature at the wall. The semi-empirical expression (6-8) agrees well with Jenkins' theory in the limit of small friction, all sliding $(\mu \rightarrow 0, r \rightarrow \infty)$. There, Jenkins predictably obtains $S / N=\mu$. Figures $(2-5)$ illustrate the fit provided by Eqs. (6-7).

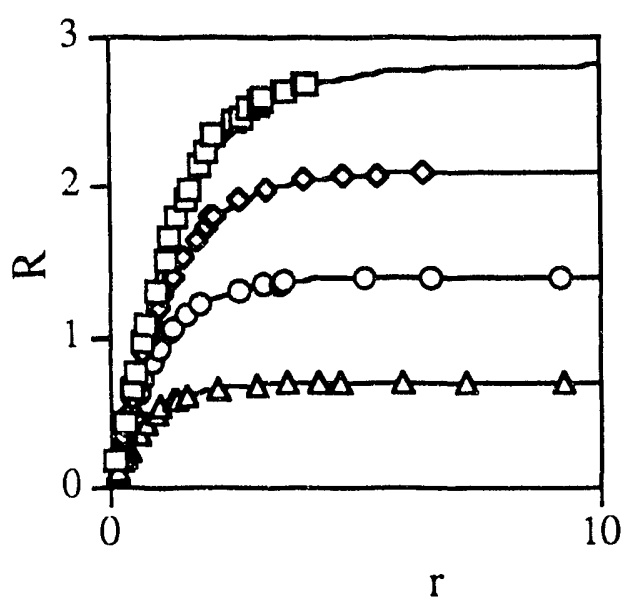

Fig. 2. Effective friction for $e=1$. The triangles, circles, diamonds and squares represent simulations results for $\mu=0.1,0.2,0.3$ and 0.4 , respectively. The lines correspond to the best fit of Eqs. (6-7). By definition, $R \equiv(7 / 2)(1+e) /\left(1+\beta_{0}\right) S / N$.

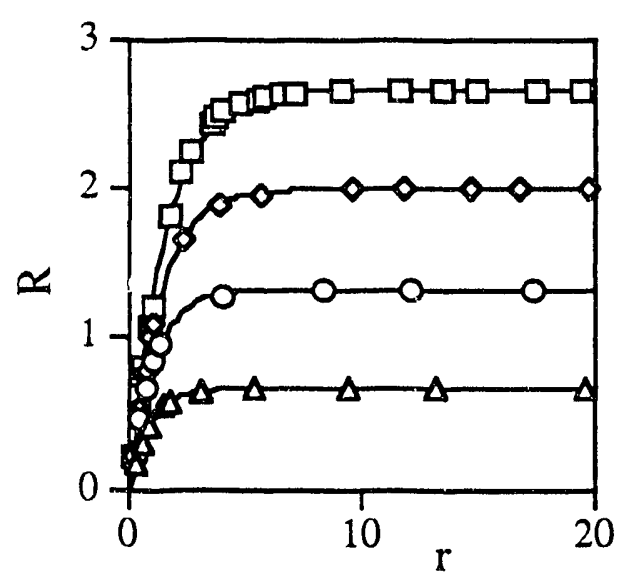

Fig. 3. Effective friction for $\mathrm{e}=0.9$. The symbols have the same meaning as in Fig. 2. 


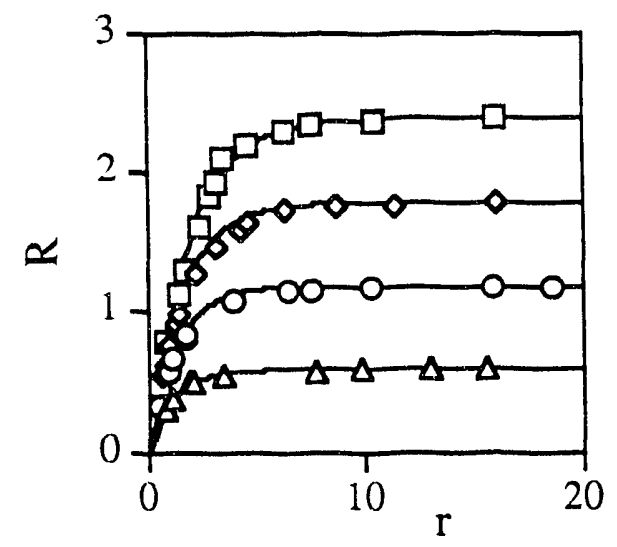

Fig. 4. Effective friction for $e=0.7$. The symbols have the same meaning as in Fig. 2.

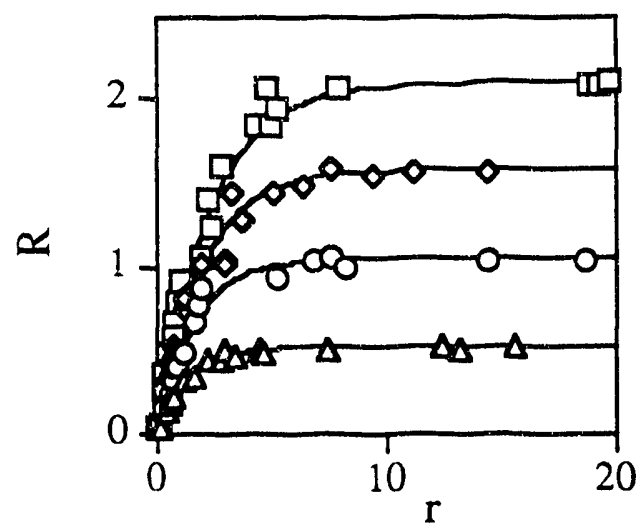

Fig. 5. Effective friction for $e=0.5$. The symbols have the ame meaning as in Fig. 2 .

For $0.5 \leq e \leq 1$ and $0.1 \leq \mu \leq 0.4$, we find that the tanger tial restitution coefficient $\beta_{0}$ has a small effect on $S / N$. Figure 6 illustrates the dependence of $S / N$ on $\beta_{0}$.

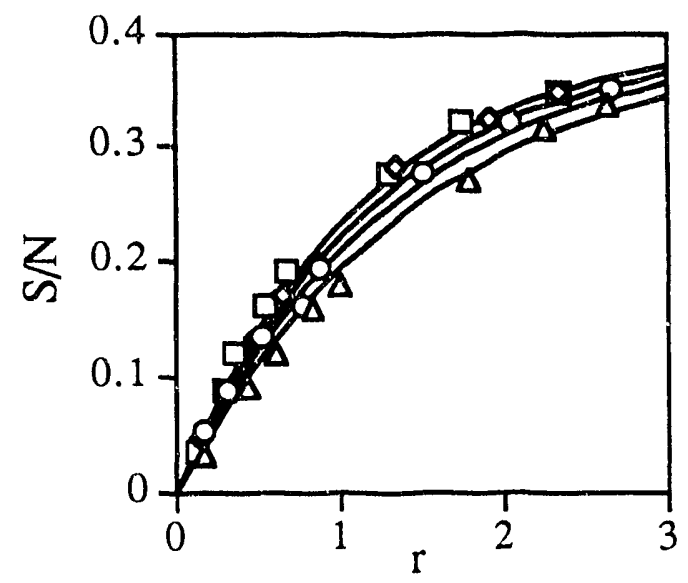

Fig. 6. $S / \mathrm{N}$ for $\mathrm{e}=0.9, \mu=0.4$. The triangles, circles, diamonds and squares are $\beta_{0}=0,0.2$, 0.4 , and 0.6 , respectively. The lines correspond to the best fit of Eqs. (6-7). 
The flux of fluctuation energy $Q$ added to the flow by the wall has two limits. The first corresponds to low sliding $(r \rightarrow 0)$. Here we find

$\frac{\mathrm{Q}}{\mathrm{N} \sqrt{3 \mathrm{~T}}} \approx \frac{0.05}{1+\mathrm{e}} \mathrm{r}^{2}+0.52 \mu(1-\mathrm{e}) \mathrm{r}-\mathrm{c}$,

where

$\mathrm{c}=\mathrm{A}\left[1-\mathrm{e}^{-\mathrm{S}} \mu\right]$,

$\mathrm{A} \approx 1.79(1-\mathrm{e})^{2}+1.11(1-\mathrm{e})+0.1$,

$\mathrm{S} \approx 4.64(1-\mathrm{e})+8.60$.

The second limit corresponds to all sliding $(r \rightarrow \infty)$. Here, we obtain

$\frac{\mathrm{Q}}{\mathrm{N} \sqrt{3 \mathrm{~T}}} \approx 0.522(1+\mathrm{e}) \mu^{2}+2.57(1-\mathrm{e}) \mu-1.08(1-\mathrm{e})$.

Figures (7-9) illustrate the resulting fit for $Q$ in the two limits above.

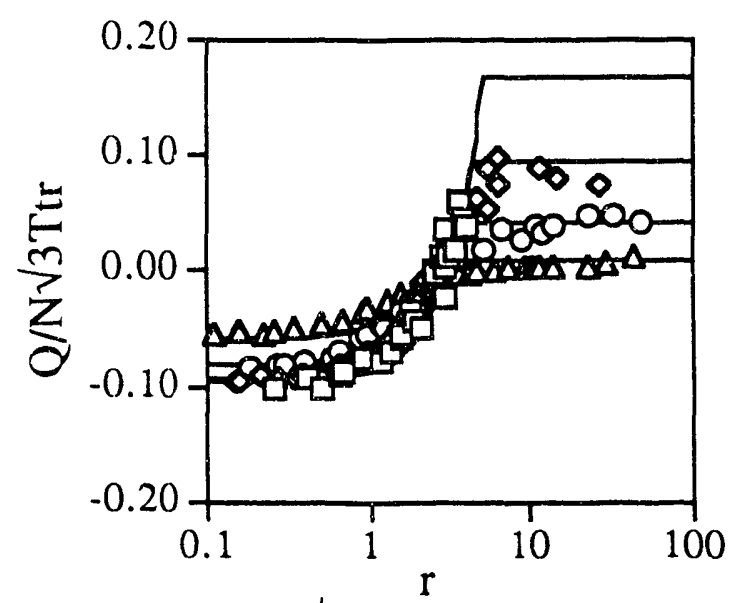

Fig. 7. Relative granular energy flux $\mathrm{Q} / \mathrm{N} \sqrt{ } 3 \mathrm{~T}$ for $\mathrm{e}=1$. The triangles, circles, diamonds and squares represent simulations results for $\mu=0.1,0.2,0.3$ and 0.4 , respectively. The lines correspond to the best fit of Eqs. (9-13).

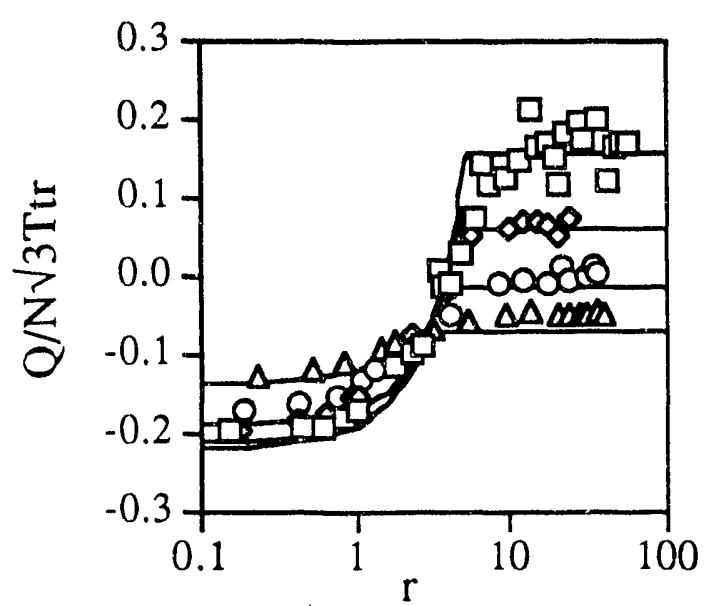

Fig. 8. Relative granular energy flux $\mathrm{Q} / \mathrm{N} \sqrt{ } 3 \mathrm{~T}$ for $\mathrm{e}=0.9$. Symbols are found in Fig. 7. 


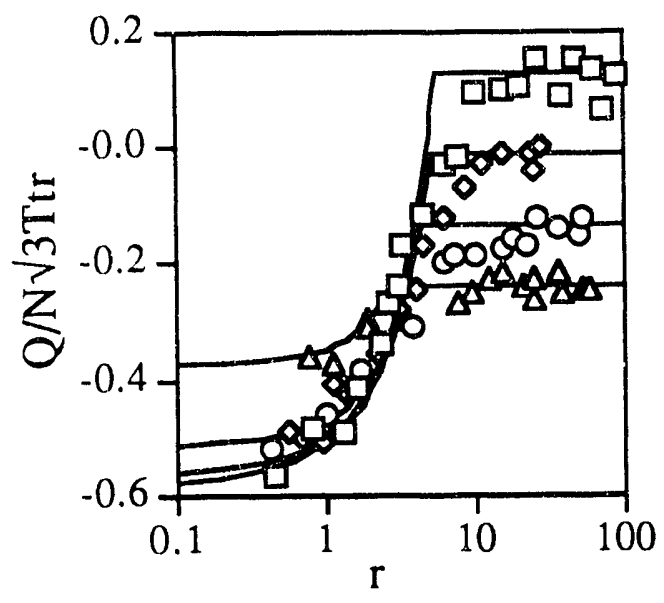

Fig. 9. Relative granular energy flux $\mathrm{Q} / \mathrm{N} \sqrt{ } 3 \mathrm{~T}$ for $\mathrm{e}=0.7$. Symbols are found in Fig. 7. 

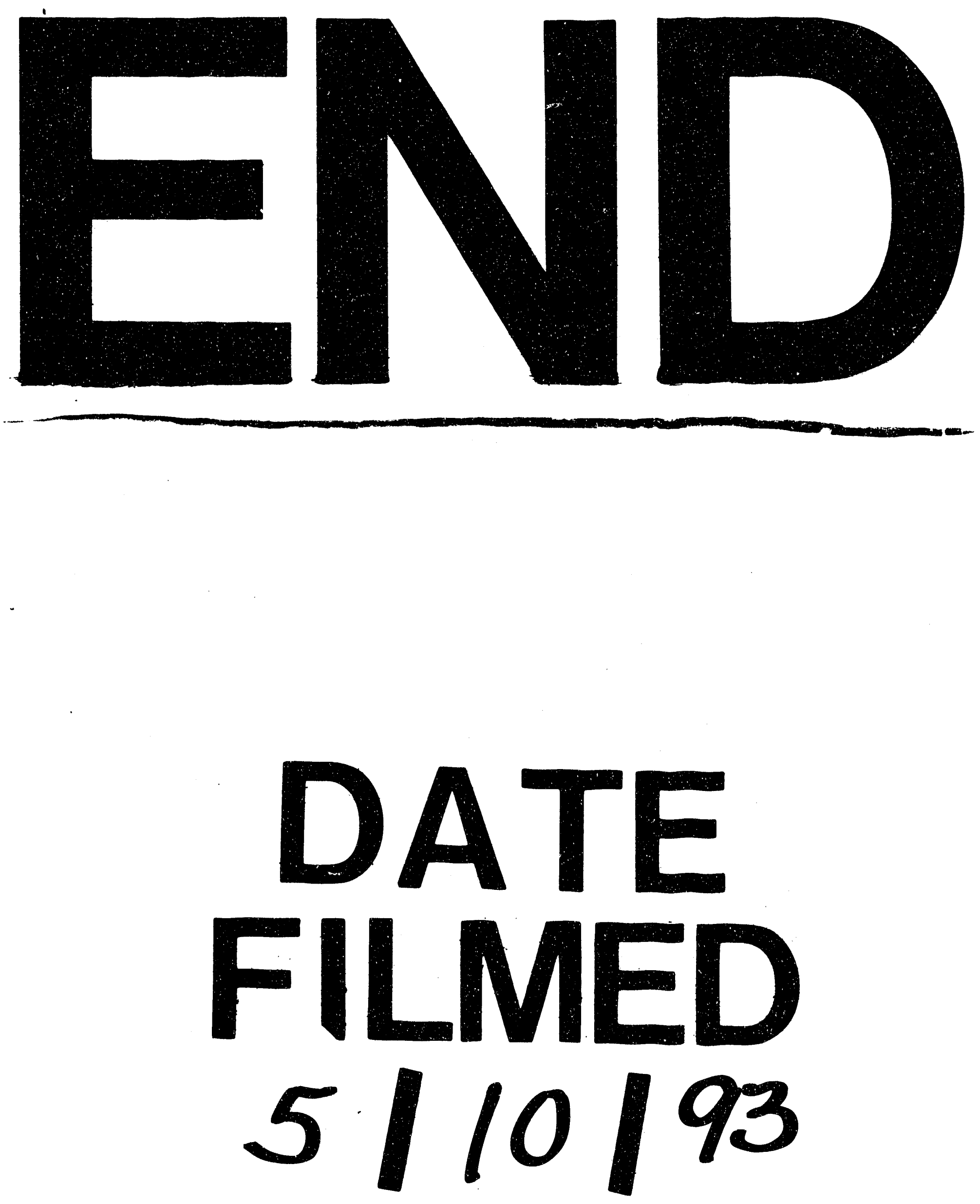

1 
I 